\title{
La teatralità del disumanar nell' Inferno dantesco sullo sfondo della "vocazione drammatica" di Dante
}

\author{
Maria Maślanka-Soro \\ Uniwersytet Jagielloński w Krakowie \\ maria.maslanka-soro@uj.edu.pl
}

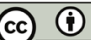

\section{Riassunto}

In questo articolo intendo proporre, da un lato, una breve sintesi relativa al problema della teatralità nella Divina Commedia, e dall'altro mi soffermo ad analizzare più dettagliatamente questo fenomeno in uno degli episodi più spettacolari dell'Inferno, quello delle metamorfosi dei ladri. Il discorso sulla dimensione teatrale rimane nel presente articolo in funzione di altri discorsi (filosofico, teologico), coerentemente all'intentio auctoris. Riferendomi all'ilemorfismo aristotelico considero il contrapasso metamorfico dei dannati come triste conseguenza del danno arrecato dal peccato mortale alla loro virtus informativa.

Parole chiave: Dante; teatralità; vocazione drammatica; disumanar; metamorfismo; ilemorfismo aristotelico.

\begin{abstract}
What I seek to propose in this article is, on the one hand, a brief summary about the issue of theatricality in the Divine Comedy; on the other hand, I seek to analyze this phenomenon in greater detail in one of the most dramatical episodes of Inferno, that of the thieves' metamorphoses. The discourse on theatrical dimension remains in this article in relation to other discourses (philosophical, theological), consistent with the intentio auctoris. Referring on Aristotelian hylomorphism, I consider the metamorphic contrapasso of the damned as a sad consequence of the damage caused by mortal sin to their virtus informativa.
\end{abstract}

Keywords: Dante; theatricality; dramatic vocation; disumanar; metamorphism; Aristotelian hylomorphism. 
Ciovanni di Salisbury considera la vita umana uno spettacolo teatrale in I cui l'uomo recita la parte che gli è estranea, illudendosi di avere la libertà di pensare ed agire: "Comoedia est vita hominis super terram, ubi quisque sui oblitus personam exprimit alienam" (Polycraticus, III, 8, in PL, t. I99, col. 488). Il dramma umano con l'avvento della morte cede però lo spazio alla tragedia: "rerum omnium tragicus finis est" (ivi, col. 49I). Già nella tarda antichità Epitteto, partendo da simili presupposti, constatava non proprio ottimisticamente che dagli uomini - attori nel theatrum mundi della vita dipende interpretare bene il personaggio nel quale si devono immedesimare, ma la scelta di esso spetta non a loro, ma a qualcun altro (Enchiridion, XVII).

Dante-poeta probabilmente non avrebbe condiviso queste opinioni, vista l'importanza che dava al libero arbitrio sulla cui concezione poggia la struttura etica e teologica della sua Commedia ${ }^{\mathrm{I}}$. È da notare, invece, come questo pensiero rimanga in sintonia con la condizione dei dannati nell'Inferno che sono privi di quella libertà di spirito di cui godevano, assieme ad altri, prima di abbandonare le loro "spoglie" (If. XIII, IO3) ${ }^{2}$. Ora la desolata esistenza che conducono è circoscritta - a ben vedere, sia nel senso letterale che allegorico, entrambi ritenuti veri ${ }^{3}$ dall'autore - allo spazio di un girone e sospesa nell'eterno presente, dove è incessantemente "attualizzata" nei loro ricordi, di cui si servono in maniera arbitraria, sempre a proprio favore ${ }^{4}$, nonché nelle emozioni violente e senza sbocco, con cui cercano di aggrapparsi alla "vita lieta" (If. XIX, v. IO2)5, definitivamente perduta da questa "perduta gente" (If. III, 3 ).

A nessun lettore sfugge che l'impressionante visione dantesca dell'aldilà, coerente nell'intento generale con il pensiero cristiano, obbediente nei minimi

I. Com'è noto nell'Epistola XIII, 7-8, la definizione del senso allegorico, comprensivo di altri due sensi, anagogico e morale, è basata sul libero arbitrio. Ė superfluo ricordare che la questione del libero arbitrio viene discussa da Marco Lombardo nella parte centrale della Commedia (Pg. XVI).

2. Tutte le citazioni della Commedia nel presente articolo provengono dall'edizione: Alighieri I994.

3. È ben noto che Dante suggerisce (nell'Epistola XIII) l'applicazione alla sua opera della cosiddetta allegoria dei teologi sul modello della esegesi biblica; cfr. almeno Singleton 1978: 32-36; II5 sgg. Nella Commedia più di una volta (soprattutto negli ultimi canti del Purgatorio) il poeta allude al suo carattere veritiero (cfr. ad es. Pg. XXXII, I03-IO5; XXXIII, 52-54; 79-8I; Pd. IV, I24-I25: "Io veggio ben che già mai non si sazia / nostro intelletto, se 'l ver non lo illustra") e si ritiene uno ispirato direttamente da Dio (cfr. ad es. Pd. XXV, I-2).

4. Uno dei migliori esempi della manipolazione degli eventi passati da parte di un dannato viene fornito da Ugolino della Gherardesca nel suo lungo racconto (If. XXXIII, 22-75) in cui egli espone la sua tragedia, tacendo alcuni particolari che lo potrebbero compromettere agli occhi di Dante-personaggio, ma viene smascherato da Dante-autore, soprattutto nella "cornice" narrativa, dove si manifesta il suo "bestial segno".

5. L'epiteto "lieta" spetta qui alla vita terrena, ma potrebbe alludere anche alla condizione paradisiaca inaccessibile ai dannati, visto che la esprime in diversi altri luoghi della Commedia (cfr. ad es. If. VII, 95; Pg. V, 46; XXIV, I4; XXXI, I27; Pd. III, 68; V, 94; XV, 67; XXVII, IO4). 
particolari alla potente immaginazione del genio poetico dell'Alighieri, pur appartenendo al genere epico, è altamente drammatica e spettacolare, indipendentemente - com'è risaputo - dal suo titolo che nella mente dei lettori medievali non evocava però le stesse idee e concetti che, infatti, nella totale o parziale ignoranza riconosciuti dallo stesso Sommo Poeta nell' Epistola XIII, Io (ammettendo che sia sua), di che cosa fosse il genere comico vero e proprio, con le regole elaborate dagli antichi, lo consideravano tuttavia molto capiente. A conferma di tale giudizio mi sia lecito riportare le considerazioni di Zygmunt Barański:

It is possible to show that, of all the medieval modi, comedy was the most elastic and wide-ranging. Thus, it also accommodated characters and feelings of every kind [...] it had links with prose [...] and it employed a wealth of formal registers [...] Comedy embraced every subject and style; it could signify literature in general, not least since in the Middle Ages it had lost its dramatic associations [...] The term also indicated the poem's religious dimension. St Jerome had noted comedy's moral force (Ep. 54, 9), while scriptural sermo humilis [...] had ties with the 'comic' stilus humilis in the thirteenth century, like comedy, the Bible was quoted as an example of both the 'low' and 'middle' style (Barański 1997: 13-I4).

Grazie a questo stile "misto" ossia il "pluralismo stilistico" (Contini 1970: I7I), la Commedia è in grado di esprimere ogni sfumatura della condizione umana - nonostante il poeta non nasconda le difficolta cui va incontro, trattandosi di una materia mai affrontata prima nella sua pienezza ${ }^{6}$ - dall'estrema miseria morale del "vermo in cui formazion falla" (Pg. X, I29) alla più alta sublimità dell' "angelica farfalla" (Ivi, I25). Una volta tolte le barriere stilistiche, si apre la via alla mescolanza dei generi letterari che fu per molte generazioni - fino alla nostra - fonte di irrepetibili emozioni estetiche e non solo tali. Infatti, nonostante qualche tentativo passato di operare una distinzione tra "poesia" e "struttura", destinato a fallire, ormai, come dimostrano ancora di più le ricerche degli ultimi anni, non sussistono dubbi sul valore poetico del pensiero dantesco, come pure sulla perfetta corrispondenza tra esso e la ricchissima sfera dell'elocutio. Se è difficile non concordare con l'osservazione di un critico (Perrus 2000: 436) che il vero protagonista di un testo letterario è il linguaggio, rimane altrettanto vero che Dante, oltre ad essere capace di esprimere ogni cosa, riesce a piegare la lingua del suo poema in modo da superare i confini tra le arti: penso a numerosi passi in cui egli avvicina la sua attività di scrittore-scriba Dei (Pd. X, 27: "quella materia ond'io son fatto scriba") $)^{8}$

6. Cfr. ad es. If. XXXII, I-I2 e Pd. II, I-I5, in particolare il v. 7: "L'acqua ch'io prendo già mai non si corse".

7. Alludo ovviamente alla teoria crociana (Croce I92I) e ai suoi pochi seguaci.

8. Cfr. anche Guido da Pisa (1974: Introduzione, in http://dante.dartmouth.edu/search. php): "Re vera, potest ipse dicere verbum prophete dicentis: "Deus dedit michi linguam 
quella di pittore che "con essempro pinga" (Pg. XXXII, 67) e che addirittura riesce a trasformare nel "visibile parlare" (Pg. X, 95) i bassorilievi scolpiti dalla "mano" di Dio nella cornice dei superbi, o a quella di coreografo e musicista, quando - nella terza cantica - fa accompagnare l'etterna letizia delle "luci sante" Pd. VII, I4I) di balli e suoni che superano di tanto le più belle melodie mai udite sulla terra ${ }^{9}$. Insomma, l'attività artistica di Dante-poeta tende soprattutto nel Paradiso - all'integrazione delle arti.

Forse ancora di più si nota la sua vocazione drammatica ${ }^{\mathrm{IO}} \mathrm{o}$ drammaticoteatrale che gli fa assumere il ruolo di regista, il quale dispiega davanti agli occhi del lettore - trasformatosi momentaneamente in spettatore, grazie alla spettacolarità di alcuni episodi che ricordano microstrutture teatrali - un "nuovo ludo" (If. XXII, II8), ricco di elementi farseschi, una "sacra rappresentazione" (Canti XXIX-XXXII del Purgatorio) o uno squarcio drammatico di teatralità gestuale e di parola, rappresentata dalla rissa verbale tra maestro Adamo e Sinone (If. XXX, IOo-I29), volgare e vivace. Oppure una tragedia mancata, "recitata" da qualche "grande" personaggio infernale che ricorre abilmente all'uso retorico della parola.

Tibor Wlassics, esaminando le strategie narrative nella Commedia, dedica un capitolo alle sceneggiature dantesche (Wlassics 1975: 183-204), dove tra l'altro nota la frequente caratteristica dell'arte del suo autore di "sceneggiare invece di spiegare" o di raccontare attraverso il dialogo (Ivi: I88). Un esempio particolarmente carico di espressività con cui vorrei illustrare quest'ultima tecnica teatralizzante, il quale non compare tra quelli esaminati dal critico, mi sembra quello della riscrittura dell'episodio virgiliano della morte di Amata (Aen. XII, 595-607). Dante opera un adattamento "scenico" di una sequenza puramente narrativa, anche se dotata di un alto grado di drammaticità. La tragedia della regina che si dà la morte credendo - delirante (v. 6or: "demens") - che Turno sia già stato ucciso da Enea e che la figlia debba andar sposa al vincitore, è stata tradotta dal "narrato in parlato" (Spera 20IO: II3) o, ricorrendo all'espressione dantesca soprammenzionata, in "visibile parlare", grazie all'amplificazione della "parte" affidata a Lavinia, personaggio muto in Virgilio. Nell'episodio che fa parte degli esempi di ira punita e si presenta

eruditam"; et illud: "Lingua mea calamus scribe velociter scribentis". Ipse enim fuit calamus Spiritus Sancti, cum quo calamo ipse Spiritus Sanctus velociter scripsit nobis et penas damnatorum et gloriam beatorum" (data d'accesso: aprile 20I4). Cfr. almeno Sarolli (1963).

9. Cfr. ad esempio la descrizione dei giri di danza che nel cielo del Sole gli spiriti sapienti compiono - cantando - intorno al punto dove sta Dante con Beatrice: Pd. X, 70-8I; XIII, I6-2I. A questo proposito ha scritto recentemente Chiara Cappuccio (Cappuccio 2008).

Io. Prendo in prestito questa espressione da Tibor Wlassics (Wlassics I975: I88). Ci sono vari studi relativi alla dimensione drammatica e teatrale di Dante; a titolo di esempio cito: Spitzer (1965); Musa (1974); Wlassics (1975); Bosco (1977); Iannucci (1983); Picone (1989); Borsellino (I99I); Bonino (I992); Maślanka-Soro (20I0); Spera (20I0). 
come una visione estatica, essa, in una "recita" teatrale, piangendo la rovina della madre le rimprovera dolorosamente di essersi lasciata dominare da una ira mala ${ }^{\mathrm{II}}$ nel timore di perdere la figlia che ora ha perduto per sempre con la morte:

surse in mia visione una fanciulla piangendo forte, e dicea: "O regina, perché per ira hai voluto esser nulla?

Ancisa t'hai per non perdere Lavina; or m'hai perduta! Io son essa che lutto, madre, a la tua pria ch'a l'altrui ruina". (Pg. XVII, 34-39)

Un'altra tecnica altamente drammatica e teatrale presente nella Commedia è quella - segnalata da Wlassics - di sciogliere la descrizione o narrazione in una esclamazione drammatica. Essa si osserva nei casi in cui una data storia (che, possiamo aggiungere, costituisce a volte una narrazione di secondo grado, in quanto si tratta della riscrittura di un mito mutuato da Virgilio o - più spesso - da Ovidio) viene colta nel suo momento più drammatico. Tale sarebbe l'episodio che fa parte di una similitudine nel Canto XVII dell'Inferno in cui la paura di Dante-pellegrino, seduto sul dorso di Gerione ${ }^{\mathrm{I} 2}$, viene paragonata a quella di Icaro prima della fatale caduta; la breve narrazione viene inaspettatamente conclusa con la drammatica esclamazione di Dedalo, comprendente pure un giudizio morale (un'altra novità rispetto ad Ovidio) ${ }^{13}$, rivolta al figlio che sta volando troppo in alto: "Mala via tieni!" (If. XVII, III). Un grado più alto di tensione teatrale si coglie, a mio avviso, laddove - nella prima cantica, particolarmente ricca in tensioni di vario genere, per lo più negative - la microstruttura teatrale fa parte di una similitudine di tipo omerico, con il comparante cioè che si allarga ad una narratio o descriptio drammatizzata. Lo possiamo illustrare ricorrendo ad esempio alla prima parte della lunga, doppia reminiscenza mitologica che apre sotto forma di similitudine "per superamento"I4 il Canto XXX dell'Inferno. Gli esempi dell' insania mentale ivi riportati devono aiutare il lettore ad immaginarsi per analogiam (ma

II. L'ira mala che fa deviare la ragione viene contrapposta da Tommaso d'Aquino all'ira bona (o ira per zelum): cfr. Tommaso d'Aquino, Summa theologiae IIa IIae, q. I58, a. I, in http:// www.corpusthomisticum.org/iopera.html (data d'accesso: aprile 20I4).

I2. Il ruolo di Gerione potrebbe essere paragonato a quello di una macchina teatrale nel teatro antico la cui funzione era tra l'altro quella di rendere possibile la discesa di una divinità dal cielo in terra. Va da sé che la rassomiglianza di queste funzioni non è effetto di un procedimento mimetico.

I3. Per una analisi dettagliata del rifacimento dantesco di questo mito cfr. Picone (2005: I40-I45).

I4. In questo genere di similitudini il comparante terreno che dovrebbe illustrare il comparato ultraterreno risulta inadeguato e viene superato negli aspetti che stabiliscono una relazione di somiglianza tra i due elementi della similitudine. Con questo procedimento iperbolico il poeta aggiunge alle funzioni della similitudine quelle dell'iperbole; cfr. Ledda (2008: 96). 
anche per antithesin) la rabbia demoniaca dei falsificatori di persone ${ }^{15}$. Danteautore evoca unicamente - com'è una prassi abituale nel suo trattamento delle favole mitiche - il momento culminante della storia che coincide con una tragedia. La follia di Atamante, il quale nella sua mente, colpita dall'insania da una divinità, trasforma in belve la moglie e i figli, sfocia in un disastro: in un rabbioso delirio egli uccide Learco sbattendolo contro la roccia e provoca la morte della moglie Ino che sfuggendo alla furia omicida del marito si getta in mare con l'altro figlio. L'esclamazione, che - questa volta - non manca neanche in Ovidio ${ }^{16}$, il cui racconto (Met. IV, 5I2-529) costituisce la fonte diretta del proemio in questione, nella versione dantesca diventa però più concisa $\mathrm{e}$ concentrata su due azioni ("tendiamo [...] pigli"), quindi più drammatica ${ }^{17}$, anche nel senso etimologico della parola:

Nel tempo che Iunone era crucciata per Semelè contra 'l sangue tebano, come mostrò una e altra fiata,

Atamante divenne tanto insano, che veggendo la moglie con due figli andar carcata da ciascuna mano, gridò: "Tendiam le reti, sì ch'io pigli la leonessa e 'leoncini al varco"; e poi distese i dispietati artigli,

prendendo l'un ch'avea nome Learco, e rotollo e percosselo ad un sasso; e quella s'annegò con l'altro carco [...]

Ma né di Tebe furie né troiane si vider mäi in alcun tanto crude, non punger bestie, nonché membra umane,

quant 'io vidi in due ombre smorte e nude, che mordendo correvan di quel modo che 'l porco quando del porcil si schiude. (If. XXX, I-I2; 22-27)

La reminiscenza mitologica è, inoltre, particolarmente ricca di verbi, il che aumenta la teatralità della scena, rendendola assai movimentata e accrescendo la velocità con cui si susseguono le azioni violente di Atamante che si illude

I5. Dato che questa similitudine anticipa la rappresentazione della furia rabbiosa nella bolgia e acquista il suo pieno significato solo retrospettivamente, la sua funzione è prolettica; cfr. Bloome (1989: 407).

I6. Ovidio, Met. IV, 5I3-5I4: "io, comites, his retia tendite silvis! / hic modo cum gemina visa est mihi prole leaena" " $\mathrm{Su}$, compagni, stendete le reti in questi boschi! Poco fa qui ho visto una leonessa con due cuccioli"; citazione originale e trad. it. dall'edizione: Ovidio 2009).

17. La radice greca $d r a-$, su cui viene costruito il verbo drao, il nome dramma ecc., appartiene al campo semantico legato all'azione. 
di dominare "la scena" volendo cacciare con la rete gli "animali" mentre in realtà è lui stesso cacciato dalla divinità. L'ironia tragica che ricorda - grazie anche al contesto morale in cui appare - l'ironia tragica nei drammi sofoclei. In più, la storia mitica subisce nella riscrittura dantesca una certa metamorfosi (e anche per questo diventa più teatrale), legata alla persona del protagonista. A differenza di Ovidio, il poeta italiano fa vedere la perdita della ragione in termini di imbestiamento che subisce il re tebano nel momento dell'omicidio, distendendo "i dispietati artigli".

Nell'accostamento delle follie antiche ${ }^{\mathrm{I}}$ a quelle che toccano ai "suoi" dannati, l'Alighieri ribadisce, difatti, tramite la forma negativa della similitudine ("né di Tebe furie né troiane"; v. 22), ciò che le differenzia: neanche i più violenti casi di follia di cui si legge nei poemi antichi, permettono al lettore di immaginarsi appieno la furia bestiale dei falsari, in quanto la realtà infernale supera in negativo il male vissuto o immaginato dagli uomini di tutti i tempi.

Rispetto, tuttavia, alla rabbia dei falsificatori di persone che corrono alla cieca e si mordono a vicenda, tra i quali viene nominata esplicitamente la Mirra "scellerata" e il "folletto" Gianni Schicchi, Atamante è vittima di una forza superiore, quella di Giunone, che a lui e alla moglie Ino aveva riservato una terribile punizione, vendicandosi, per la terza volta ${ }^{\mathrm{r} 9}$, del tradimento (l'ennesimo della serie) di Giove con la sorella di Ino, Semele. Lui e Ino pagano quindi per una "colpa" che non gli appartiene. La differenza maggiore e semanticamente più pregnante riguarda appunto la causa della follia, totalmente opposta nei due casi. Visti in questa prospettiva Atamante e Ino si presentano come personaggi tragici la cui sorte può suscitare pietà (in quanto vengono atrocemente puniti dagli dei pur essendo innocenti; cfr. Aristotele, Poet. I3, I453a 4$)^{20}$ e paura (perché sono simili a noi nella loro condizione

18. Prescindo qui dalla follia di Ecuba, meno "teatrale", ma altrettanto interessante, essendo effetto di un dolore insopportabile che alla fine sfocia in una aggressività paragonabile per il suo carattere atroce a quella di Atamante. Dante non fa apertamente nessun cenno sulla sua vendetta compiuta su Polimestore, traditore e uccisore del figlio Polidoro, ma allude direttamente alla sua metamorfosi, effetto dell'imbestiamento interiore, che tuttavia non è totale come in Ovidio, perciò più credibile e - inoltre - più simile a quella del re tebano: il lettore è tacitamente invitato ad immaginarsi che anche le sue dita, per poter ricavare gli occhi a Polimestore, si fossero dovute trasformare in "dispietati artigli". Non finiscono qui le somiglianze con il caso di Atamante, se consideriamo che in una prospettiva più ampia Ecuba è vittima di una forza maggiore che fece cadere Troia: in Dante cambia "solo" la ragione di questa caduta rispetto alla tradizione letteraria antica, poiché egli la lega alla superbia punita di cui la città di Priamo sarebbe diventata simbolo (significativa è la sua presenza tra le "figure esemplari" sulla terrazza dei superbi: Pg. XII, 6I-63), analogamente ad alcune città veterotestamentarie contaminate da una grave colpa, come Sodoma e Gomorra.

19. Giunone ha già sfogato in precedenza la sua ira contro le discendenti della casa reale di Tebe, sorelle di Ino: Agave e Semele. Dante vi allude nel v. 3 dello stesso Canto XXX dell'Inferno.

20. Qui e nella nota successiva mi riferisco all'ediz.: Aristotele (2002). 
umana; cfr. ivi, I453a 4-5). Se sulla loro storia fosse incentrata una tragedia greca, la katharsis aristotelica, che interessa prima di tutto lo spettatore, sarebbe stata possibile solo a condizione che la si intendesse (come vogliono alcune delle più convincenti interpretazioni - cfr. ad es. Somville I975: 92-96) come fenomeno estetico: lo spettatore, coinvolto nella vicenda fino a provare entrambi i sentimenti, dopo essersi reso conto del suo carattere fittizio, si libera alla fine da pietà e paura, trovando un conforto psichico ed emotivo. Tibor Wlassics nella sua brevissima menzione di questo passo dantesco gli attribuisce una forza tragica eschilea. Ma io direi, invece, che ci sono più somiglianze con il tragico sofocleo (più apprezzato, tra l'altro, da Aristotele), sia per la sostanziale "non colpevolezza" dei suoi eroi ${ }^{21}$, che per l'ironia tragica e la scelta del momento con cui si apre l'azione delle sue pièces: nella maggioranza dei casi (a giudicare dalle opere giunteci per intero) si tratta di quello immediatamente precedente la "catastrofê".

Tornando alla follia dei falsificatori di persone, essa è al tempo stesso affine e lontana da quella mitica, siccome funge da strumento della giustizia divina nei confronti di chi sfruttò la ragione per agire in malafede.

A differenza della trama di una tragedia che è solo una fictio, ciò che Dante-personaggio vede in questa e in tutte le altre bolgie, nonchè altri luoghi di pena, è, conformemente all' intentio auctoris ${ }^{22}$, reale e non finto, come si è già sottolineato. I falsari vengono - verso la fine del lungo proemio - paragonati, in forte contrasto con la sequenza mitologica, ai porci usciti dal porcile, in una similitudine che si innesta sulla precedente e che non è "negativa", ma del tutto "positiva". Questa volta l'immagine fungente da termine di paragone, fortemente sprezzante, in stile comico, che rimane in antitesi con quello tragico dell'altra, affonda le sue radici, a quanto pare, nella parabola evangelica dei porci indemoniati che precipitano nel mare trovandovi la morte (Mt 8, 28-32 $)^{23}$. La conseguenza più grave dell'abuso del dono della ratio è stata per Gianni Schicchi, Mirra ed i loro simili, la perdita della natura umana, che nel momento della morte fisica, coincisa con quella spirituale, si è manifestata tramite una metamorfosi avente un legame allusivo con la natura stessa del peccato. In realtà la transformatio dell'anima con la successiva perdita della parte divina $^{24}$ (quella che distingue un essere umano da altri esseri viventi), è

2I. Mi sia lecito ricordare qui il caso simile a quello di Atamante, di Aiace nella tragedia omonima, dove l'eroe greco viene colpito dall'insania da Atene senza essere colpevole e, per difendere il proprio onore, commette il suicidio.

22. Sull'importanza dell' intentio auctoris nella letteratura ed esegesi medievale, cfr. Minnis (1984: capp. 3 e 4).

23. L'ha notato Claude Perrus: cfr. Perrus (2000: 427).

24. Essa corrisponde alla cosiddetta transformatio moralis, a distinzione dalla transformatio supernaturalis, operata dalla volontà superiore, che si esprime con il contrapasso (e quindi comprende sia il senso letterale che allegorico). Per questa terminologia cfr. Guthmüller (200I: 6I-77). 
avvenuta ancora prima di morire, nella loro "vita lieta" (If. XIX, IO2), perciò Vanni Fucci potrà dire di sé: "Vita bestial mi piacque e non umana [...] son Vanni Fucci / bestia, e Pistoia mi fu degna tana" (If. XXIV, I24-I26). E non si tratta minimamente di un'autoaccusa, perché nell'Inferno la volontà dei peccatori è mutilata nella stessa misura che l'intelligenza e la memoria; infatti, privi di libero arbitrio (in quanto rivolti al male) loro sono costretti a obbedire alla volontà divina ${ }^{25}$, come tutti i demoni. Occorre precisare che la metamorfosi postuma riguarda il cosiddetto "corpo aereo" 26 che ogni anima crea intorno a sé grazie alla propria "virtù informativa" $(P g$. XXV, 4I). Essendo quest'ultima venuta meno in un'anima dannata che - a causa del male verso il quale si era rivolta - aveva danneggiato la sua parte razionale (Ginsberg 1991: 208), in particolare l'intelletto, non riesce mai a "formare" il corpo aereo che perfettamente rispecchi la sua figura terrestre ${ }^{27}$ : esso risulta, invece, difettoso o addirittura diventa l'immagine della "matta bestialitate" di un determinato peccatore $^{28}$, rinnegando la sua humanitas ${ }^{29}$.

Se consideriamo, da una parte, che secondo Dante ( $C v$. II, xiii, 6) tutti i dannati hanno perso il "ben de l'intelletto" (If. III, ı8), cioè la verità ${ }^{30}$ che avvicina l'uomo a Dio ${ }^{3 \mathrm{I}}$, e che - dall'altra - il concetto latino di falsitas presuppone proprio la corruzione della verità o della vera natura di una cosa (cfr.

25. Una prova lampante ne abbiamo più avanti, quando Vanni Fucci, interrogato da Dantepellegrino sulla natura della sua colpa, ammette: "io non posso negar quel che tu chiedi" (If. XXIV, 136).

26. Il meccanismo della sua formazione viene spiegato, com'è noto, da Stazio nel Canto XXV del Purgatorio nell'ambito della storia dell'anima razionale dalla sua comparsa nel corpo umano fino alla sorte postuma; cfr. ad esempio vv. 88-96: "Tosto che loco lí la circunscrive, / la virtú formativa raggia intorno, / cosí e quanto ne le membra vive. / E come l'aere, quand'è ben pïorno, / per l'altrui raggio che 'n sé si reflette, / di diversi color diventa adorno; / cosí l'aere vicin quivi si mette / e in quella forma ch'è in lui suggella / virtüalmente l'alma che ristette". Nella sua "teoria" del corpo aereo Dante utilizza, come nota Zygmunt Barański, diverse fonti simbolico-esegetiche e mistiche (cfr. Barański 200I: 403 sgg.) ed inoltre si richiama probabilmente anche all'autorità di Tommaso d'Aquino e, più esattamente, alle sue considerazioni concernenti il "corpo" degli angeli: "Dicendum quod licet aer, in sua raritate manens, non retineat figuram, neque colorem; quando tamen condensatur, et figurari et colorari potest, sicut patet in nubis. Et sic angeli assumunt corpora ex aere, condensando ipsum virtute divina, quantum necesse est ad corporis assumendi formationem" (Summa theologiae, I, q. 5I, art. 2, ad 3, in http://www.corpusthomisticum. org/iopera.html, data d'accesso: maggio 20I4).

27. Il peccato mortale può essere considerato, infatti, "failure of form" (Ginsberg I99I: 206).

28. Cfr. Pg. XXV, I06-IO7: "Secondo che ci affliggono i disiri / e li altri affetti, l'ombra si figura".

29. Dante nel Convivio (III, vi, II-I2) sostiene, seguendo l'autorità di Aristotele, che l'anima, "l'atto" del corpo e la sua "cagione", è responsabile della somiglianza dell'essere umano al suo Creatore; cfr. anche Pd. VII, 79-80: "Solo il peccato è quel che la disfranca / e falla dissimile al sommo bene".

30. Cfr. Tommaso d'Aquino, Sententia libri Ethicorum, lib. VI, lectio 3, n. 2, in http://www. corpusthomisticum.org/iopera.html: "falsum, quod est malum intellectus, sicut verum est bonum ipsius" (data d'accesso: maggio 20I4).

3I. Cristo nel Vangelo di Giovanni $(\mathrm{I} 4,6)$ dice di essere "via, et veritas, et vita" (cito da: Bibbia 1966). 
Lucchesi I99I: I07-I26), non è difficile capire perché tutti i falsari in questa bolgia (oltre ai falsificatori di persone vi "soggiornano" quelli di parola, arsi da una "febbre aguta", alchimisti e falsatori di moneta) sono afflitti da malattie più o meno ripugnanti. Nella scienza medica del Medioevo la malattia viene definita appunto come alterazione o corruzione degli umori del corpo (cfr. Perrus 2000: 426) e ciò spiega bene il ragionamento dantesco in riferimento a questi dannati.

Nell'Inferno si tratta sempre di un contrapasso che - per lo più tramite una specifica configurazione del corpo aereo - simboleggia la colpa e ne risulta una imago: Dante-pellegrino "guardandolo da spettatore" dovrebbe capire - nell'intenzione di quel regista che è Dante-autore, ma, secondo l'intentio auctoris, Dio stesso - fino in fondo il suo significato per vivere una katharsis, analoga, ma al tempo stesso antitetica a quella che Aristotele nella Poetica (naturalmente non conosciuta da Dante) considera il fine ultimo della tragedia (6, I449b: 27-28). Le tragedie nel regno di Lucifero sono, però, a differenza di quelle evocate dallo Stagirita, solo apparenti, in quanto viste come tali unicamente da alcuni peccatori mortali che si comportano come attori, indossando una specie di maschera (ovviamente tragica) e cercando, con vario successo, di suscitare pietà in Dante-personaggio. Lo possono fare, però, solo in maniera "controllata" da Dante-autore, il quale prima o poi li smaschera mettendo a nudo la verità che traspare dalle loro parole il cui pieno significato rimane a loro - che vissero in menzogna - in parte celato. Perciò i discorsi di questi peccatori fungono apparentemente da autoapologie (come ad esempio nel caso di Francesca, Pier della Vigna, Ugolino), mentre si trasformano in autoaccuse senza che loro ne siano consapevoli ${ }^{2}$. I dannati danteschi non meritano pietà (come più di una volta viene ribadito da Virgilio) e ciò rimane in pieno accordo con il pensiero neotestamentario: basta ricordare quei luoghi dove Cristo accenna alla pena eterna. Uno dei commentatori danteschi, Guido da Pisa, ne parla a conclusione delle sue riflessioni, osservando che Cristo si dimostrò misericordioso nei confronti di Maria Maddalena, ma non provò alcun sentimento simile per le anime destinate all'inferno: "In isto enim mundo est tempus misericordie; in alio, autem, est solum tempus iustitie" ${ }^{33}$. All'uscita da

32. I casi di Francesca, Pier della Vigna, Brunetto Latini ed Ugolino sono i più lampanti, ma pure Farinata e Ulisse possono essere visti - a primo approccio - come personaggi tragici, assomiglianti agli eroi sofoclei a causa della loro magnanimità che inizialmente non lascia capire i valori in cui credono come negativi o insufficienti alla salvezza. Per le analisi più approfondite della loro (e di altri personaggi) apparente tragicità cfr. Maślanka-Soro (I999: I83-I94; 2004: I04-II8; 20II: 609-633). Siccome loro, come tutti gli altri dannati nell'Inferno dantesco, hanno perso in questa vita il "ben de l'intelletto", cioè la verità (cfr. If. III, I8), nell'altra continuano a vivere nella menzogna. Per il problema del tragico nella Commedia cfr. Maślanka-Soro (20Iob).

33. Guido da Pisa (1974): commento a If. XX, 28-30; http://dante.dartmouth.edu/search.php (data d'accesso: marzo 20I4). 
alcuni gironi, non solo dell'Alto Inferno, Dante-pellegrino si libera dalla pietà sorta in lui alla vista della miseria in cui sono sprofondati post mortem anche quelli che durante la vita avevano avuto qualche merito: basti pensare agli "abitanti" del Limbo, a Farinata, Brunetto Latini o ai suoi compagni di pena, quelli "ch'a ben far puoser li 'ngegni" (If. VI, v. 8I). Ciò conferma la comprensione della natura del male da parte di Dante-personaggio ed è la condizione sine qua non della sua crescita spirituale. La katharsis ha quindi il carattere conoscitivo ed etico, ma non estetico (contrariamente a come avviene nelle tragedie antiche, prima di tutto quelle greche), perché si tratta di una realtà "vera", non fittizia. Non bisogna poi dimenticare che il cammino attraverso il primo luogo ultraterreno viene definito all'inizio della prima cantica come "guerra / sì del cammino e sì de la pietate" (If. II, 4-5), vale a dire in termini di conflitto, non irrisolto, bensì tale che dovrà essere superato dal viaggiatore.

La teatralità presente a livello di elocutio (non solo nelle similitudini, ma anche nelle citazioni o allusioni letterarie di vario genere) rimane in funzione degli episodi infernali che l'immaginazione del poeta, sorretta dal pensiero biblico, teologico e letterario, dispiega davanti agli occhi di Dante-pellegrino, trasformandoli a volte in piccoli spettacoli con microsequenze di gesto e di parola. A volte, però, il protagonista del viaggio viene coinvolto da uno spirito dolente nel dramma che in qualche modo riguarda anche lui (come nel primo incontro infernale, con Francesca da Rimini), oppure nel quale viene implicato dagli stessi dannati che cercano di far sorgere in lui dei sentimenti da loro desiderati, ad esempio la già menzionata pietà o ammirazione e stima; così si comportano Pier della Vigna, Farinata degli Uberti o Brunetto Latini ${ }^{34}$, i quali in un primo momento riescono nei loro intenti, perché il protagonista è lacerato da un contrasto tra il loro valore individuale (morale, civile, intellettuale) e l'atto peccaminoso passibile di dannazione.

Nei casi sopra accennati l'aspetto scenico, quello che impegna di più la vista, rivela (come in tutti gli altri incontri nel primo regno ultraterreno) la materializzazione della colpa ed è dialetticamento opposto alle "parole ornate" (If. XVIII, 9I $)^{35}$, al loro uso retorico e persuasivo da parte di un determinato peccatore che cerca a volte di sfruttare a suo favore la metamorfosi subita, come nella famosa constatazione, lamentevole e accusatoria al tempo stesso,

34. Sono cose ben note, perciò mi permetto di farvi un semplice cenno.

35. Virgilio usa questa espressione per caratterizzare la retorica di Giasone che gli permise di sedurre Isifile. Forse non è casuale che la stessa espressione in singolare si riferisce alla poesia di Virgilio (If. II, v. 67) nel discorso di Beatrice (da lui stesso riportato) che aveva come obiettivo convincere Dante-pellegrino della volontà divina che stava dietro il suo viaggio ultraterreno e la scelta della guida. Nell'uno e nell'altro caso credo che si tratti del potere persuasivo di un discorso poetico o comunque retoricamente ricercato, il quale di per sé non è mai neutro, ma sempre o positivo, o negativo, in quanto strettamente connesso con l'oggetto della persuasione. 
di Pier della Vigna: "Uomini fummo, e or siam fatti sterpi" (If. XIII, 37). La "recita" con le parole si rivela efficace fino al momento in cui non diventa compromettente nel suo centrale discorso, dove rivela, tra l'altro, la sua etica "paganeggiante" (Maślanka-Soro 20Iob: 249-255). In ogni caso essa contribuisce a ritardare la comprensione (da parte di Dante-pellegrino e del lettore implicito) del senso e grado della contaminazione del male in cui il dannato è immerso. L'effetto finale (con la katharsis di cui si è già parlato) risulta quello di una tragedia mancata.

Laddove Dante-pellegrino ha a che fare con i colpevoli dell'uso distorto della ragione, il che si manifesta con la distruzione quasi totale dell' imago Dei nella parte razionale dell'anima (l'unica capace di attuare la perfezione della specie umana e di renderla simile al Creatore), l'aspetto esteriore degli "abitanti" dell' Inferno svolge un ruolo importante nella loro "recita".

Uno dei casi più interessanti - dal punto di vista della teatralità - del disumanar ${ }^{36}$ ossia imbestiamento ${ }^{37}$, è indubbiamente quello dei ladri. Per di più, le loro metamorfosi fanno da spettacolo non solo a Dante e Virgilio, ma agli stessi dannati (come vedremo) che in ogni momento possono aspettarsi di diventare vittime di una simile trasformazione. Lo spettacolo, "ideato" e "diretto" da Dio, è per il pellegrino che - non occorre dimenticarlo - rappresenta l'umanità sulla via della conversione, "una lezione magistrale" sulla condizione sprituale dei "sommersi" (If. XX, v. 3). Il ruolo di Dante-poeta sarebbe quello di uno scriba divino che traduce in parole-segni ciò che ha visto di persona: la vera essenza della natura dei peccatori, resa un "visibile parlare" grazie al meccanismo del fallimento dell'anima soprammenzionato, incapace - per il danno riportato dalla sua parte razionale - di formare la materia conformemente alla propria natura.

La "recita infernale" in questa bolgia ${ }^{38}$ si svolge in "tre tempi" in cui "gli spettatori" assistono a tre diverse "esibizioni" delle anime dei ladri attraverso le quali si manifesta la giustizia divina. L'ironia dell'invenzione dantesca consiste nel fatto che gli stessi dannati fungono da suoi strumenti, perché i ser-

36. Francesco da Buti inventò il termine disumanar come antitetico al neologismo dantesco trasumanar: cfr. Francesco da Buti (I858-1862): commento a Pd. I, 64-72, in http://dante. dartmouth.edu/search.php: "Che li omini scelerati che sono rifiutati da Dio si disumanano e diventano bestie varie, secondo vari vizi, come dice ancora Boezio nel predetto luogo nel libro terzo, e diventano sozzi et oscuri quanto all'anima, come è lo dimonio, stando in questa vita" (data d'accesso: marzo 2014).

37. Interessanti sviluppi sul tema dell'imbestiamento nella bolgia dei ladri offre, in un contesto più ampio di confronto tra il metamorfismo dantesco e quello dannunziano, il saggio di Marino Balducci (Balducci 2006).

38. Dante-autore sottolinea il suo carattere disumano chiamandola con una parola fortemente sprezzante - zavorra (cfr. If. XXV, I42: "Così vid'io la settima zavorra / mutare e trasmutare") che è un hapax legomenon. Questo termine ribadisce la degradazione di quelli la cui materia corporale ha perso l'identità a favore di uno strano ibridismo tra umano e serpentino; cfr. anche Alighieri (20II): commento ad locum. 
penti - aggrovigliandosi in gran numero sul fondo della settima bolgia - non sono altro che i ladri momentaneamente metamorfizzati. Nella scelta del serpente l'immaginario dantesco si rifà alla cultura cristiana (colta e popolare), dove questo animale simboleggia la fraudolenza maligna. Inoltre, viene sfruttata l'analogia tra l'arte del latrocinio e il costume del serpente, accomunati dall'astuzia ingannevole, rapidità silenziosa delle mosse e abilità nel celarsi.

Se ci riferiamo alla concezione aristotelica dell'ilemorfismo, il furto momentaneo del corpo (ossia della materia) da parte di un essere a cui i ladri assomigliano di più, indica la loro doppia natura ed è una conseguenza logica della perdita delle facoltà appartenenti alla ratio e alla sua virtus informativa, in seguito ad un peccato mortale.

Nel primo degli episodi che ci interessano (If. XXIV, 97-II7) Vanni Fucci, uno dei ladri fiorentini più malfamati dell'epoca ${ }^{39}$, attaccato da un serpente (sotto le cui spoglie si cela l'anima di un altro ladro) si incendia, brucia e diventa cenere che in un istante subisce un processo inverso, provocando in lui una grande confusione ed angoscia, come in un indemoniato o epilettico a cui viene paragonato in una doppia similitudine (vv. II2-II8). L'incenerimento e la successiva "rigenerazione" hanno punti di riferimento più eruditi: Dante-autore fonde il modello lucaneo con quello ovidiano delle Metamorfosi, cioè la transformatio naturalis di un milite di Catone Uticense, provocata dal morso di un serpente e descritta naturalisticamente (Phars. IX, 762-788), con la morte e rinascita della fenice (Met. XV, 39I-407).

L'uccello miracoloso che nel racconto di Ovidio muore nel suo nido fatto di erbe aromatiche, per poi rinascere ad una nuova vita, altrettanto lunga e straordinaria, nella letteratura teologica medievale diventa simbolo della risurrezione e del trionfo della vita sulla morte. Nulla di strano che nella iconografia cristiana rappresenta la figura di Cristo (Kobielus 2002: 98). Nel paragonare la morte e la rinascita del ladro e della fenice, il tertium comparationis ossia l'analogia risulta ambigua, in quanto Vanni Fucci risorgerà dalle ceneri non per una nuova vita ma per la stessa morte spirituale in cui rimane fin dal suo 'piovere' "in questa gola fiera" (If. XXIV, I23). È facile immaginarsi - data la ripetitività di tutti gli eventi nella prima regione oltremondana, da intendersi forse come la loro assurda moltiplicazione nell'eterno presente infernale - che anche questa metamorfosi è sottoposta ad un ritmo ciclico. Se ciò è vero, allora si potrebbe dire che Vanni Fucci e i suoi compagni di pena a cui tocca questo genere di contrapasso, soffrono ciclicamente una distruzione totale dell'anima che potrebbe prefigurare il suo stato di non-essere

39. Vanni Fucci fu, come rilevano alcune fonti medievali (Anonimo fiorentino, Storie pistoiesi ecc.), famoso per la sua violenza e la sua crudeltà gratuita che gli aveva meritato l'appellativo di "bestia". Come ladro si specializzava nel depredare chiese; il furto più famoso fu quello degli oggetti sacri appartenenti alla sagrestia di san Iacopo nel Duomo di Pistoia. 
dopo il Giudizio Universale ${ }^{40}$, coincidente con la seconda morte ${ }^{4 \mathrm{I}}$. Con ciò si spiegherebbe lo smarrimento e la grande angoscia che egli prova dopo la "risurrezione".

Stravolgendo il senso del modello Dante-poeta ne opera una parodia: invece dello spettacolo che presenta lo schema vita-morte-vita, ne crea uno che raffigura sempre e solo la morte, nelle sue - per così dire - diverse sfumature, tra prima e seconda morte dell'anima, con la prospettiva di una fusione in un incendio apocalittico (sottinteso) ${ }^{42}$ che coinvolgerà tutti gli spiriti destinati all'"etterno essilio" (If. XXIII, I26) ${ }^{43}$.

Prima della metamorfosi successiva (If. XXV, 49-78) ossia della seconda "recita" in questa bolgia, si fa sentire la voce dell'autore, il quale assume il ruolo di spettatore che ha assistito ad una scena inaudita e quindi poco credibile. La strategia retorica che serve a rinforzare la veridicità del racconto, consiste nell'assicurare i suoi lettori - in una apostrofe diretta - che neanche lui avrebbe creduto in quello che sta per descrivere, se non l'avesse visto con i propri occhi:

Se tu se' or, lettor, a creder lento ciò ch'io dirò, non sarà maraviglia, che io che 'l vidi, a pena il mi consento. (If. XXV, 46-48).

La teatralità della drammatica narrazione che segue, viene messa in un maggior rilievo tramite il ricorso ad un pubblico composto di due dannati (Buoso Donati e Puccio Sciancato), i quali - a differenza di Dante-pellegrino e Virgilio - commentano stupiti un orrendo processo metamorfico che accade sotto i loro occhi: "Omè, Agnel, come ti muti! / Vedi che già non se' né due né uno" (vv. 68-69).

È la reazione alla vista di una fusione in corso tra Agnolo Brunelleschi e un serpente in cui si era trasformato nel frattempo Cianfa Donati. Si potrebbe interpretarla, a mio avviso, come una metamorfosi "guastata", nel momento in cui l'anima di Brunelleschi ha perso completamente il controllo sopra la materia che le appartiene ed ha subito una trasformazione davvero mostruale. La forma venuta fuori, assurda e difficile da immaginarsi, ma soprattutto da definire, descritta dai due ladri-spettatori come "né due né uno" (v. 69), e dal narratore più avanti come "due e nessun" (v. 77), potrebbe essere intesa come

40. Cfr. le parole di Farinata (If. X, IO6-I08) che svelano a Dante-pellegrino la condizione delle anime dannate: "Però comprender puoi che tutta morta / sia nostra conoscenza da quel punto / che del futuro sia chiusa la porta".

4I. Cfr. If. I, II7. Agostino descrive la seconda morte come un perenne status moriendi: cfr. De civ. Dei XIII, II; ma cfr. anche Ap 20, I4.

42. L'Apocalisse di san Giovanni parla dello stagno del fuoco, dove verranno gettati tutti quelli che non figureranno nel "libro della vita": cfr. Ap 20, II-I5.

43. Cfr. anche $P g$. XXI, I8. 
una perversa ${ }^{44}$ antitesi della doppia natura di Cristo, al tempo stesso "due e uno" (Beal I983: III; Gross 1985: 66) ${ }^{45}$. Avremmo qui la parodia blasfema di uno dei principali dogmi cristiani. Non è la prima blasfemia in questa bolgia ${ }^{46}$.

La fonte dantesca della trasfigurazione di cui stiamo parlando, taciuta da Dante-autore che invece si richiama più avanti - apparentemente senza motivo ${ }^{47}$ - alla metamorfosi di Aretusa, è, com'è risaputo, il mito ovidiano della trasformazione della ninfa Salmacis e di un giovane di nome Ermafrodito (Met. IV, 365-379), divenuti un essere bisessuale. Alcuni calchi verbali e dei motivi simili non lasciano dubbi sul modello intertestuale (cfr. Hawkins I99r: 22 , nota 8 ), ma anche in questo caso (come nel precedente) assistiamo ad una antitesi all'interno di un rapporto analogico. La ninfa, innamorata follemente del giovane Ermafrodito sfuggente al suo amore, lo costringe alla fine ad un atto sessuale che si conclude inaspettatamente per entrambi con la sopraddetta metamorfosi. Cianfa-serpente, invece, diviene strumento di punizione nei confronti di Agnolo e il suo atto di mordere le guance di quest'ultimo (che inizia il processo della fusione e corrisponde - perversamente - ai baci di Salmacis) è dettato non dall'amore, ma dall'odio accompagnato dall'invidia.

La terza metamorfosi, più complessa delle precedenti, preceduta dalla sfida emulativa di Dante-autore nei confronti di Lucano e Ovidio ${ }^{8}$, ma soprattutto di quest'ultimo, ritenuto nel Medioevo optimus magister transformationum $^{49}$, si presenta come un processo che si svolge in due direzioni opposte: il serpente diventa l'uomo e l'uomo il serpente. La ripresa del modello classico si manifesta solamente nella descrizione di ciò che succede a Buoso Donati e si limita ad alcuni particolari mutuati dal mito di Cadmo nel momento della sua trasfigurazione, come l'unirsi delle gambe, l'indurimento della pelle, il cadere per terra o la biforcazione della lingua ${ }^{50}$.

44. Non è casuale l'uso dell'epiteto "perversa", riferito all'effetto della metamorfosi: "due e nessun l'imagine perversa / parea" (77-78).

45. Cito entrambi gli autori dall'articolo di Bodo Guthmüller: cfr. Guthmüller (2000: 349); la bibliografia su questo canto a pp. 356-357.

46. Cfr. If. XXV, 3, dove Vanni Fucci si rivolge a Dio con una frase blasfema, accompagnata da un gesto osceno.

47. Sembra impossibile un errore da parte di Dante-autore che conosceva troppo bene le Metamorfosi di Ovidio. Forse si tratta di un gioco con il lettore che ha come obiettivo invitarlo a scoprire il "furto" dantesco in questa bolgia dei ladri: cfr. Hawkins (I99r: 22, nota 8 ).

48. "Taccia di Cadmo e d'Aretusa Ovidio, / ché se quello in serpente e quella in fonte / converte poetando, io non lo 'nvidio" (If. XXV, 97-99).

49. Benvenuto da Imola (I887), commento a If. XXV, 97-99, in http://dante.dartmouth.edu/ search.php (data d'accesso: febbraio 2014).

50. Cfr. Ovidio, Met. IV, 579-580: "commissaque in unum / paulatim tereti tenuantur acumine crura" e Dante, If. XXV, I06-Io8: "Le gambe con le cosce seco stesse / s'appicca sì, che 'n poco la giuntura / non facea segno alcun che si paresse"; Ovidio, Met. IV, 586-587: "Ille quidem vult plura loqui, sed lingua repente / in partes est fissa duas" e Dante, If. XXIV, 
La trasformazione simultanea e scambievole dei connotati più profondi dei due dannati inizia con il morso malizioso di Francesco Cavalcanti, "un serpentello acceso" (If. XXV, 83), all'ombelico di Buoso. Mentre si fissano con uno sguardo ipnotico (Ivi, 9r: "Elli 'l serpente e quei lui riguardava"), emettono l'uno dalla bocca e l'altro dalla ferita un denso fumo o vapore che si incontra e confonde, creando un'atmosfera di angoscia e di orrore. Sembra un incantesimo in cui un ruolo fondamentale spetta agli occhi fissamente puntati gli uni negli altri fino alla fine:

l'un si levò e l'altro cadde giuso, non torcendo però le lucerne empie, sotto le quai ciascun cambiava muso.

(If. XXV, I2I-I23)

La "vendetta" di Cavalcanti forse è giustificata dalle ragioni storiche di cui si legge in tutti gli importanti commenti danteschi. Nondimeno le sue parole - "I' vo' che Buoso corra, / com' ho fatt'io, carpon per questo calle" (Ivi, I4O-I4I) - solo apparentemente sono manifestazione di un libero atto di volontà. In realtà, questa bolgia, così spasimante di odio, rivela in maniera più dinamica e altamente teatrale la vera natura dei dannati. La loro cattiva volontà è una proiezione "nuda" di quella occultata durante la vita, e smaschera le loro relazioni reciproche che - qui più vistosamente che altrove nel Basso Inferno - si dimostrano come l'adempimento della loro figura terrena (Auerbach 2007: 205-226).

La rappresentazione perfettamente sincronica di questo fenomeno sovrannaturale costituisce indubbiamente una novità rispetto alle descrizioni ovidiane e perciò non fa meraviglia la decisa affermazione dantesca della superiorità della propria arte. Sono però convinta che il rapporto emulativo non si limiti, nell' intentio auctoris, contrariamente a come possiamo leggere in molti commenti antichi e moderni, all'aspetto prettamente formale. Robert Hollander è, tra pochi critici, a sostenere che la vera novità dell'arte dantesca rispetto a quella ovidiana è di "non stare inventando, ma solamente di star 'osservando' la realtà della vendetta divina sul fondo della settima bolgia” (Alighieri 20II: 208, commento ai vv. 94-IO2). Condividendo pienamente questa tesi che si iscrive nella problematica molto più ampia - del falso e vero nel poema dantesco, vorremmo in qualche modo completarla, soffermandoci sulla seconda parte dell'apostrofe metaletteraria, il cui "bersaglio" è chiaramente il solo Ovidio:

ché due nature mai a fronte a fronte non trasmutò sí ch'amendue le forme

I33-I34: “e la lingua, ch'avëa unita e presta / prima a parlar, si fende". Tutti i loci paralleli indica Paratore (1973: 229-236). 
a cambiar lor matera fosser pronte.

(If. XXV, IOо-IO2).

La terzina fa parte del discorso che riguarda la poesia in tutti i suoi aspetti, sia formali che semantici e in essa Dante-poeta alluderebbe, a nostro avviso, al preannuncio del tema principale delle Metamorfosi nel loro proemio e all'incapacità di andare oltre il sensus litteralis, tipica per Ovidio ritenuto da Dante homo rhetoricus.

Cercando di interpretare questi versi alla luce dell'ilemorfismo aristotelico che, come si è visto, ha un ruolo importante nel contrapasso dantesco manifestatosi con una trasformazione dell'anima, si può ipotizzare che se le anime infernali non hanno più intatta quella essenza che è l'anima rationalis, responsabile, tra l'altro, della formazione della materia in maniera conforme alla sua natura, devono, come le forme "difettose", entrare in nuovi corpi. Ne segue che la propositio ovidiana - "In nova fert animus mutatas dicere formas / corpora" (Met. I, I-2) acquista pieno senso solamente nelle trasformazioni dantesche. Il poeta italiano invera questa propositio, ma ciò significa che riconosce la sua inadeguatezza alle descrizioni dell'optimus magister transformationum, rifiutato in questa bolgia appunto come maestro. Secondo Dante le metamorfosi ovidiane hanno il valore retorico, ma non quello morale, filosofico e teologico. La polemica riguarda, quindi, il concetto stesso della metamorfosi. Per svolgerla egli ricorre spesso ai mezzi non verbali di comunicazione, ad una teatralità diffusa che conferma pienamente la sua vocazione drammatica.

\section{BiBLIOGRAFIA}

Alighieri, D., 1994, La Commedia secondo l'antica vulgata, a c. di G. Petrocchi, 2a edizione riv., Firenze, Le Lettere.

-, zorI, La Commedia, vol. I: Inferno, a c. di R. Hollander, traduzione di S. Marchesi, Firenze, Olschki.

Aristotele, 2002, Poetica, Introduzione, traduzione e note di D. Lanza, Milano, BUR. Auerbach, E., 2007, Studi su Dante, Milano, Feltrinelli.

Balducci, M., 2006, D'Annunzio interprete di Dante e le metamorfosi, Carla Rossi Academy (Monsummano Terme - Pistoia), Carla Rossi Academy Press http:// www.phoenixfound.it/download/NEW/44.PDF.

Barański, Z., I997, "Dante and Medieval Poetics", in Dante. Contemporary Perspectives, ed. by A.A. Iannucci, Toronto-Buffalo-London, University of Toronto Press.

—, 20oI, "Canto XXV", in Lectura Dantis Turicensis, vol. II: Purgatorio, a c. di G. Güntert e M. Picone, Firenze, Cesati, pp. 389-406.

Beal, R., 1983, "Dante among thieves: allegorical soteriology in seventh bolgia", Mediaevalia, IX, pp. IO2-I23.

Benvenuto da Imola, I887, Comentum super Dantis Aldigherij Comoediam, a cura di J. Ph. Lacaita, Florentiae, Barbèra; http://dante.dartmouth.edu/search.php. 
Bibbia, 1966, La Sacra Bibbia, traduzione dai testi originali, Roma, Edizioni Paoline.

Bloome, R., I989, "Il palinsesto della memoria. Osservazioni sulla similitudine dantesca", in Filologia e critica dantesca. Studi offerti a Aldo Vallone, Firenze, Olschki, pp. 403-4I7.

Bonino, G. D., I992, "La 'teatralità' dell'Inferno dantesco", Letture classensi, 20/2I, pp. I6I-I73.

Borsellino, N., I99I, "Ludi demoniaci in Malebolge", in Id, Sipario dantesco, Roma, Salerno, pp. 3I-45.

Bosco, U., I977, "Dante e il teatro medievale", in Studi filologici, letterari e storici in memoria di Guido Favati, a c. di G. Varanini e P. Pinagli, Padova, Antenora, I, pp. I35-I 47.

Cappuccio, C., 2008, "Strutture musicali del cielo del Sole: Dante e Beatrice al centro della danza dei beati", Tenzone, 8, pp. I47-I78.

Contini, G., 1970, Varianti e altra stilistica. Una raccolta di saggi (1938-1968), Torino, Einaudi.

Croce, B., I92I, La poesia di Dante, Bari, Laterza.

Francesco da Buti, I858-1862, Commento sopra La Divina Commedia di Dante Allighieri, ed. with Notes and an Introduction by V. Cioffari, Pisa, Fratelli Nistri; http://dante.dartmouth.edu/search.php.

Ginsberg, W., I99I, "Dante, Ovid, and the Transformation of Metamorphosis", Traditio, XLVI, pp. 205-233.

Giovanni di Salisbury, Polycraticus, III, 8, in Patrologiae cursus completus, series latina $(P L)$, ed. J.P. Migne, Paris I844, t. I99.

Gross, K., 1985, "Infernal Metamorphoses: an Interpretation of Dante's 'counterpass", Modern Language Notes, Ioo, pp. 42-69.

Guido da Pisa, 1974, Expositiones et Glose super Comediam Dantis, ed. with Notes and an Introduction by V. Cioffari. Albany, N.Y., State University of New York Press; http://dante.dartmouth.edu/search.php.

Guthmüller, B., 200o, "Canto XXV", in Lectura Dantis Turicensis, vol. I: Inferno, a c. di G. Güntert e M. Picone, Firenze, Cesati, pp. 345-367.

-, 200I, "Transformatio moralis e transformatio supernaturalis nella Commedia di Dante", in Il pensiero filosofico e teologico di Dante Alighieri, a c. di A. Ghisalberti, Milano, Vita e Pensiero, pp. 6I-77.

Hawkins, P.S., I99I, "Metamorphosis of Ovid", in Dante and Ovid. Essays in Intertextuality, edited by M. U. Sowell, Binghamton, New York, Center for Medieval and Early Renaissance Studies, pp. 17-33.

Iannucci, A. A., 1983, "Dante e il Vangelo di Nicodemo: la 'Discesa di Beatrice agl'Inferi'", Letture classensi, I2, pp. 39-6o.

Kobielus, S., 2002, Bestiarium chrześcijańskie. Zwierzęta w symbolice i interpretacji: Starożytność i średniowiecze, PAX, Warszawa, PAX.

Ledda, G., 2008, Dante, Bologna, Il Mulino.

Lucchesi, V., I99I, "Giustizia divina e linguaggio umano. Metafore e polisemie del contrapasso dantesco", in Studi Danteschi, LXIII, pp. 53-I26.

Maślanka-Soro, M., 1999, "La dimensione tragica di Farinata e Cavalcante nel Canto X dell'Inferno di Dante", in Lingua e Letteratura IV, a c. di S. Widłak, Cracovia, Universitas, pp. 183-94.

—, M., 2004, “M'insegnavate come l'uom s'etterna: la cecità tragica del ser Brunetto nel Canto XV dell'Inferno di Dante", Romanica Cracoviensia, 4, pp. IO4-II8. 
—, 20Ioa: "Les formes dramatiques et théâtrales dans la Divine Comédie de Dante", European Medieval Drama, I4, pp. I2I-I35.

—, 20Iob: Tragizm w „Komedii” Dantego, Kraków, Universitas.

—, 20II, Dante e la tradizione della tragedia antica nella "Commedia", in Lectura Dantis 2002-2009. Omaggio a Vincenzo Placella per i suoi settanta anni, a c. di A. Cerbo, Napoli, Università degli Studi di Napoli L'Orientale, Officine "GraficoEditoriali "il Torcoliere", vol. II: Lectura Dantis 2004-2005, a c. di A. Cerbo, con la collaborazione di R. Mondola, A. Žabjek e Ciro Di Fiore, pp. 609-633.

Minnis, A., 1984, Medieval Theory of Authorship: Scholastic Literary Attitudes in the Later Middle Ages, London, Scolar.

Musa, M., 1974, At the Gates of Dis, in Id. Advent at the Gates: Dante's Comedy, Bloomington and London, Indiana University Press, pp. 65-84.

Ovidio [Publius Ovidius Naso], 2009, Metamorfosi, a c. di N. Scivoletto, Torino, UTET.

Paratore, E., 1973, "Ovidio", in Enciclopedia Dantesca, vol. IV, Roma, Istituto dell'Enciclopedia Italiana, pp. 225-236.

Perrus, C., 200o, "Canto XXX", in Lectura Dantis Turicensis, vol. I: Inferno, a c. di G. Güntert e M. Picone, Firenze, Cesati, pp. 425-436.

Picone, M., 1989, "Giulleria e poesia nella Commedia: una lettura intertestuale di Inferno XXI-XXII", Letture classensi, I8, pp. II-30.

—, 2005, La riscrittura di Ovidio nella "Commedia", in P. Gibellini (a c. di), Il mito nella letteratura italiana, vol. I: Dal Medioevo al Rinascimento, a c. di G. C. Alessio, Brescia, Morcelliana, pp. I25-173.

Sarolli, G.R., 1963, Dante "scriba Dei", Torino, Società Editrice Internazionale.

Singleton, Ch.S., 1978, La poesia della "Divina Commedia", Bologna, Il Mulino.

Somville, P., 1975, Essai sur la Poétique d'Aristote, Paris, Vrin.

Spera, F., 20IO, "La drammatizzazione infernale", in Id. La poesia forte del poema dantesco, Firenze, Cesati, pp. I49-I72.

Spitzer, L., 1965, "The Farcical Elements in Inferno, Cantos XXI-XXIII", in Essays on Dante, ed. by M. Musa, Bloomington, Indiana University Press, pp. I72-I76.

Tommaso d'Aquino, Summa Theologiae, in http://www.corpusthomisticum.org/iopera.html.

-, Sententia libri Ethicorum, in http://www.corpusthomisticum.org/iopera.html.

Wlassics, T., 1975, Dante narratore, Firenze, Olschki. 
